\title{
EAI Endorsed Transactions

\section{Deterministic Scheduling for Energy Efficient and Reliable Communication in Heterogeneous Sensing Environments in Industrial Wireless Sensor Networks}

\author{
Mohsin Raza ${ }^{1}$, Hoa Le-Minh ${ }^{1}$, Nauman Aslam², Sajjad Hussain ${ }^{3}$ \\ ${ }^{1}$ Department of Math, Physics and Electrical Engineering, Northumbria University, UK \\ ${ }^{2}$ Department of Computer Science and Digital Technologies, Northumbria University, UK \\ ${ }^{3}$ School of Engineering, Electronic and Nanoscale Engineering, University of Glasgow, UK
}

\section{Abstract}

The present-day industries incorporate many applications, and complex processes, hence, a large number of sensors with dissimilar process deadlines and sensor update frequencies will be in place. This paper presents a scheduling algorithm, which takes into account the varying deadlines of the sensors connected to the cluster-head, and formulates a static schedule for Time Division Multiple Access (TDMA) based communication. The scheme uses IEEE802.15.4e superframe as a baseline and proposes a new superframe structure. For evaluation purposes the update frequencies of different industrial processes are considered. The scheduling algorithm is evaluated under varying network loads by increasing the number of nodes affiliated to a cluster-head. The static schedule generated by the scheduling algorithm offers reduced energy consumption, improved reliability, efficient network load management and improved information to control bits ratio.

Keywords: Industrial Wireless Sensor Networks (IWSNs), scheduling algorithm, time deadline, TDMA, Wireless Sensor Network (WSN), energy efficiency, reliability, heterogeneous sensing, time scheduling,

Received on 23 May 2017, accepted on 03 July 2017, published on 11 July 2017

Copyright (c) 2017 Mohsin et al., licensed to EAI. This is an open access article distributed under the terms of the Creative Commons Attribution licence (http://creativecommons.org/licenses/by/3.0/), which permits unlimited use, distribution and reproduction in any medium so long as the original work is properly cited.

doi: 10.4108/eai.11-7-2017.152764

\section{Introduction}

The industrial systems with incorporated automation and process control involves a wide variety of processes running simultaneously [1, 2]. Each of these processes depend on information sampled from a number of sensors playing critical role in the smooth operation of these processes. Depending on the significance, feedback requirements and allowable deadlines, each of these sensors may have different sampling times [3]. Therefore, sensors involved in different processes and applications, embedded in the industrial automation and feedback control systems, have very diverse update frequencies. The time deadlines of the sensors involved in the regulatory feedback, open loop control and monitoring systems vary significantly [1, 4]. Since the sensors in the industrial environments belonging to these three systems exist in the same vicinity and cannot be isolated based on geographic placement, therefore, scheduling for heterogeneous time deadlines must be performed. In typical Wireless Sensor Networks (WSNs), the communication of such diverse deadlines is mostly handled by adopting the Carrier Sense Multiple Access/ Collision Avoidance (CSMA/CA) [5, 6, 7], which allows the individual nodes to access the channel on need basis or when the deadline is approaching. However, due the lack of reliability in CSMA/CA based channel access schemes, its use is not much appreciated in IWSNs. The critical nature of the information [8] in industrial applications and severity of the consequences of failed communications, more reliable channel access schemes are preferred. Hence, the TDMA based channel access scheme serves as a more suitable alternate compared to CSMA/CA based channel access in IWSNs [9, 10]. Furthermore, high dependability of Quality of Service (QoS) on number of nodes in CSMA/CA based schemes also limits its scope for dense networks. It is for the same reasons, TDMA based channel access was introduced in 
IEEE802.15.4e, (industry oriented WSN communication standard) for improved reliability and acceptable QoS [11]. Since TDMA based channel access schemes involve prior scheduling, solutions are proposed to offer runtime schedule, which takes into account the deadlines of the affiliated nodes in every superframe and formulate a schedule for the nodes for each superframe. The runtime scheduling although offers deadline based schedule, however, due to the need for increased number of control bits (used for schedule update in every frame) information to control bits ratio is decreased. Furthermore, the affiliated nodes need to stay in active listening mode during control and schedule exchange periods to ensure if their communication is scheduled or not. Given the importance of long lifetime of the sensor nodes, afore mentioned scheduling solution degrades the battery life significantly, resulting early depletion of node's energy. Apart from this, since the TDMA based channel access schemes require prior assignment of time-slots to the network nodes, the symmetrical access of the nodes cannot always be ensured in runtime scheduling.

The shortcomings of the opportunistic approach (CSMA/CA) and runtime scheduling can be addressed with the symmetric scheduling. However, for diverse time deadlines, the formation of symmetric schedule using TDMA based channel access mechanism is a challenging task. The schedule complexity further increases with the increase in the variations in the deadlines and number of nodes to be scheduled.

In this paper, a scheduling algorithm is proposed which considers varying time deadlines for different nodes, affiliated to the cluster-head. The proposed scheme transforms the deadlines to form a deterministic schedule. The proposed algorithm reduces the overall scheduling complexity by incorporating suitable approximations to the varying deadlines. The formulated schedules using proposed scheme improves the reliability by reducing frequent changes. It also enhances the lifetime of sensor nodes by reducing the active listening periods, hence allowing them to stay in the deep sleep mode for longer duration

The rest of the paper is organized as follows. Section II presents the literature review, Section III presents the system model of the proposed scheduling algorithm. Results are presented in Section IV. Finally, Section V concludes the paper and gives the future directions.

\section{Literature Review}

Dynamic nature of the industrial processes and harsh channel conditions makes wireless communications in such networks much complex. Under such circumstances the reliability and efficient data delivery in IWSNs becomes a challenging task. Furthermore, some of the conventional communication protocols impact negatively on the battery life of the nodes eventually affecting the network lifetime. It is further assumed that battery life is not as significant as reliability and maintaining desired QoS, given the abundance of readily available power sources in industrial environments. Although, it is true for certain cases, it cannot be linearised to all the possible applications in industries and for most cases, energy efficiency plays an important role.

Guaranteed channel access in TDMA based schemes though offer improved reliability, however, the conflict-free assignment of the time-slots for the nodes in accordance with the update frequency requirements becomes much more challenging with the increase in the number of nodes. Literature, in this aspect is very limited and provides very fewer works regarding the efficient scheduling of the large number of nodes.

In [12], authors have proposed two heuristic algorithms to solve the schedule minimization problem and have ensured packet delivery. Authors have also evaluated upper bounds for these schedules as a function of total packets generated in the network. In [13, 14], authors further improved the results in [12] and showed how their work outperforms [12]. In [13], authors considered harsh dynamic environment but failed to offer guaranteed data reliability. In [14], authors improve reliability in harsh control environments and form hypergraph to increase scheduling flexibility. Moreover, two schemes are also presented in [14], namely dedicated scheduling and shared scheduling and were applied to wireless sensor and control networks for performance evaluation. Another TDMA based scheme, ShedEx is introduced in [15]. This paper extends the concept of reliability improvement by repeating most rewarding slots and presents a scheduling algorithm to guarantee certain specified reliability.

\section{System Model}

Due to a wide variety of applications in the industrial environments, the International society of Automation (ISA) has divided industrial systems in six types $[1,2]$. Each of these systems offer services for specific application areas including emergency, regulatory control, supervisory control, open loop control, alerting and monitoring. Based on the class of application and the sensitivity of the observed parameter, a deadline is usually affiliated with all sorts of periodic communications in industrial environments. As IWSNs serve as a mean to communicate the generated traffic from various sensing nodes, it should also ensure the timely scheduling of communications to avoid unwanted delays. Since the deadlines of the sensed parameters can vary significantly, from few milliseconds to even seconds and minutes, it is difficult to establish a deterministic schedule for TDMA based IWSNs, especially when it is intended to ensure timeslot assignment within the specified time deadline. Furthermore, the efficiency of the schedule decreases with the increase in the number of nodes with desynchronized deadlines.

This The proposed system uses TDMA based channel access and basic frame structure of IEEE802.15.4e, Low Latency Deterministic Networks (LLDN). The superframe structure for LLDN is represented in Figure 1. A cluster based approach is considered where star topology is implemented to connect sensor nodes with the cluster-head. The prespecified schedule is used for the TDMA based communications within the cluster with group 
acknowledgement (Gack) based confirmation and shared slots for retransmission of the communication. The communication of data to the final destination is supported by the hierarchical architecture.

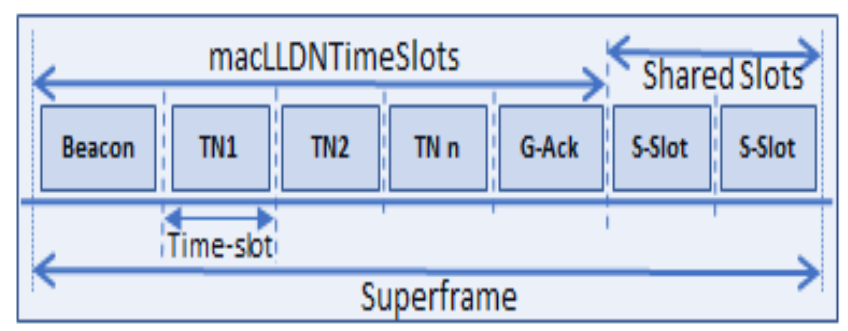

Figure 1. IEEE 802.15.4e Communication Frame structure with Group acknowledgement and shared slots

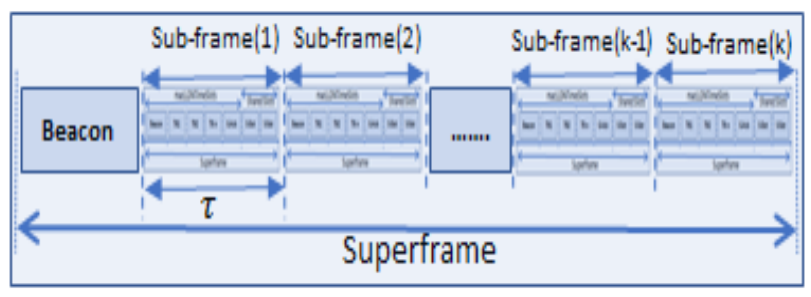

Figure 2. Superframe structure for the proposed scheduling algorithm

To schedule large number of transmitting nodes, with desynchronized time deadlines, the modified superframe is presented in Figure 2. In this case, each superframe is divided in sub-frames, each of duration $\tau$. The sub-frame replicates the IEEE 802.15.4e frame structure presented in Figure 1. The proposed superframe serves as an envelope which holds multiple sub-frames. The duration of the superframe is controlled by the lowest sampling frequency $\left(f_{\min }\right)$ of all the nodes in cluster and is given by $T_{s f}=1 / f_{\min }$. The duration of the sub-frame, $\tau$ is given by: $\tau=\frac{T_{s f}}{s f \text { Count }}$, where $s f$ Count is the number of sub-frames in a superframe. Sub-frame duration can also be represented as: $\tau=1 / f_{\text {max }}$, where $f_{\text {max }}$ is the frequency of the most frequently communicating node. The total transmissions that can take place during a superframe are denoted by $n$ and is presented in equation 1 .

$$
n=k \times \frac{T_{s f}}{\tau}
$$

Here $k$ is the number of time-slots per sub-frame (see Figure 1).

In any possible scenario, the total time slots required for proper communication of every node in the cluster is defined by the number of nodes in a cluster and time delay between two consecutive transmissions i.e. frequency of transmission of the nodes. A generalized equation for total required transmission slots, $\sigma$, is presented in equation 2 .

$$
\sigma=\frac{\left(\sum_{i=1}^{S} f_{-} b_{i t} \times s_{i} \times S y m\left(r_{i}, \quad s y m_{-} d l\right)\right) \times T_{s f}}{B_{\Delta}}
$$

Here $s$ is the total number of sources, $f_{-} b i t s_{i}$ is the number of bits to be transmitted by the source $i$ in every communication, $r_{i}$ is the sampling rate of the node $i, B_{\Delta}$ is the number of bits communicated in single transmission and sym_dl is the vector of all possible symmetric deadlines for the values of $T_{s f}$ and $\tau$. The $\operatorname{Sym}\left(r_{i}, s y m_{-} d l\right)$ ensures the symmetrical attributes of the superframe by approximating $r_{i}$ to the nearest symmetrical deadline from the $s y m_{-} d l$ vector. The symmetrical deadline selected is either lower than or equal to $r_{i}$ to ensure the in-time delivery of the information from all the affiliated nodes.

To evaluate the scheduling efficiency of the algorithm, overall load on the network is also evaluated. Here the Network Load (NL) is defined as the ratio of the required transmission slots $(\sigma)$ to the total available slots $(n)$ and is presented in equation 3 .

$$
N L=\frac{\sigma}{n}
$$

The information provided in equation 3 gives a measure of the network load handled by the algorithm and saturation point of the algorithm.

The communication between the cluster-head and the source nodes is established in two phases. In first phase (setup phase) the nodes send request to affiliate with a cluster-head depending on the received RSSI values. The request message also includes the data requirements of a particular node, specifying the details of number of transmission slots needed per unit time. Once the requests are received the cluster-head acknowledges the affiliation of the nodes to the cluster. It is assumed that the load on the cluster-head is shared with the neighboring clusters if the data requirements for all the requests approach to the maximum load limitation of the cluster-head (i.e. $\sigma \rightarrow n$ ). Along with the confirmation of the affiliation to a cluster, the cluster-head also assigns localized identification numbers to the nodes. Once the nodes are associated to the relevant cluster-heads, each cluster-head broadcasts a communication schedule. In the second phase (data communication phase), based on the shared schedule the transmission of the sensed data from the sensor nodes to the cluster-head takes place. Any new nodes trying to affiliate with the cluster-head after the setup phase use control channel. The affiliation is provided based on the availability of vacant transmission slots for communication of upcoming node. 


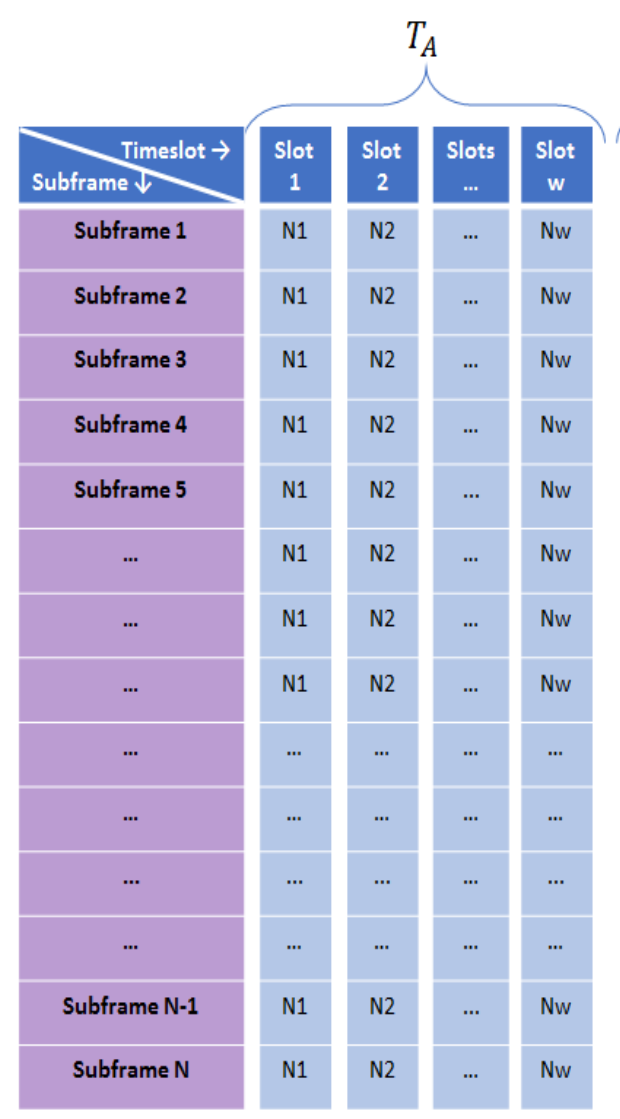

Unscheduled slots

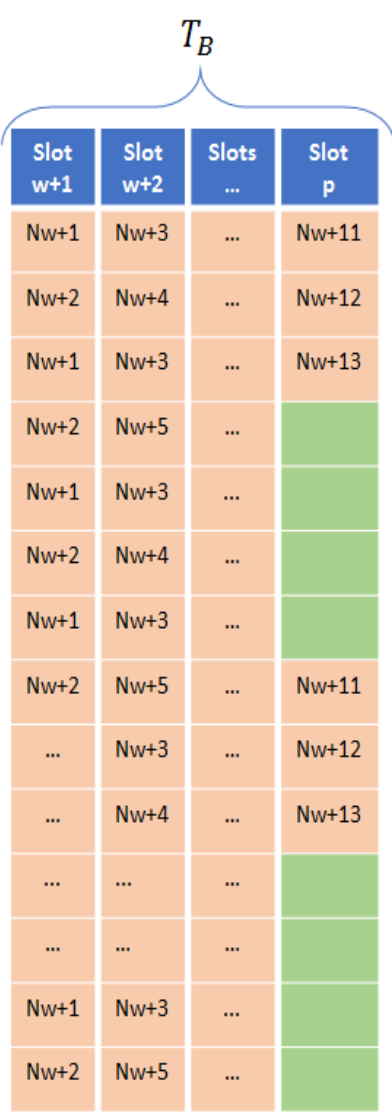

Traffic Type $T_{B}$ scheduled

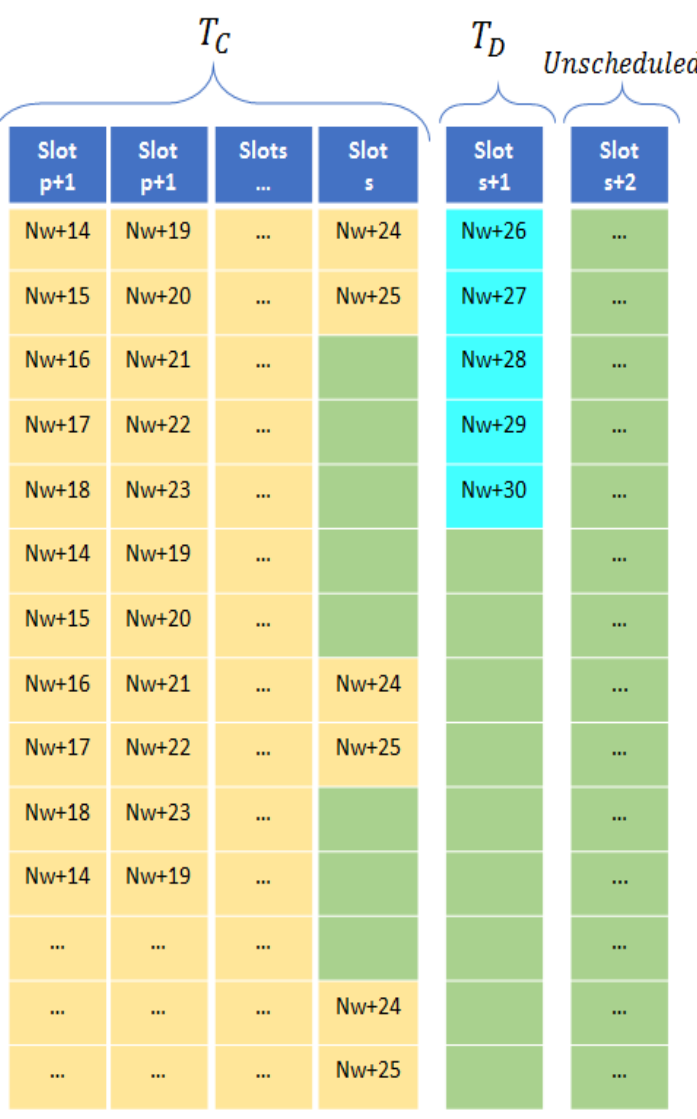

Traffic Type $T_{C}$ scheduled

Traffic Type $T_{D}$ scheduled

Figure 3. Superframe communication schedule for nodes affiliated to cluster-head

The communication schedule evaluated at the cluster-head based on the received transmission requirements of the nodes provides a static schedule, which requires no modifications unless a new node sends affiliation request to the cluster-head or association status of one or more associated nodes is changed over time. In addition, with the new association or disassociation of nodes, the schedule of the already affiliated nodes does not change, enabling the undisturbed nodes' operation for longer duration. Two primary benefits are achieved due to this deterministic schedule:

(i) Improved reliability due to less frequent changes in the nodes' transmission schedule and stability in communications

(ii) Improved energy efficiency due to the extended sleep periods and reduced idle listening periods

As stated earlier, the sensed information from different nodes with communication frequencies ranging from $f_{\min }$ to $f_{\text {max }}$, define the duration of superframe $\left(T_{s f}\right)$ and subframes $(\tau)$. Furthermore, $T_{s f}=N \times \tau$, is also ensured where $N$ is positive integer.
The scheduling of heterogeneous sensing deadlines is considered highly complex; therefore, some nonconventional means are proposed for the efficient schedule generation. Since the target application areas for the proposed scheme are regulatory feedback, open loop control and alerting traffic, therefore, all the nodes needs to be scheduled can be categorized as periodic communication sources.

To reduce the scheduling complexity, the sources in the IWSNs are divided into four categories, purely based on their communication deadlines. These categories include:

(i) $T_{A}$ : sources with communication frequency, $f_{\text {max }}$, which communicate during every sub-frame i.e. in every $\tau$.

(ii) $T_{B}$ : sources communicating once every $(2 \times Q) \times \tau$ intervals (where $Q$ is a positive integer and $Q<N / 2$ ) This category deals with nodes with time deadlines which are even multiples of $\tau$.

(iii) $T_{C}$ : sources communicating in every $((2 \times Q)+1) \times \tau$ intervals (where $Q$ is a positive integer and $Q<$ $N / 2$ ). This category deals with the nodes having time deadlines as odd multiples of $\tau$. 
(iv) $T_{D}$ : sources with communication frequency, $f_{\text {min }}$, which communicate once every superframe.

The total number of source nodes in each category $T_{A}, T_{B}$, $T_{C}$ and $T_{D}$ are $\mathrm{w}, \mathrm{b}, \mathrm{c}$, and $\mathrm{d}$ respectively. The scheduler first schedules $T_{A}$ then $T_{B}$ then $T_{C}$ and at last $T_{D}$. The scheduling algorithm is presented in Algorithm 1, whereas an example scenario for the scheduling is presented in Figure 3.

Based on the transmission requirements from all the affiliated nodes to the cluster-head, a transmission schedule is defined for a period of $T_{s f}$ (the duration of the superframe). The same schedule is repeated in every superframe. A map of the superframe schedule is presented in Figure 3. As it can be seen in the figure, each row shows the nodes (where $\mathrm{N} 1$ to $\mathrm{Nw}+30$ are node IDs) scheduled in a particular sub-frame and columns represent the time slots in each sub-frame where one time slot can facilitate communication of one sensor node. Since $T_{A}$ represents the nodes which communicate in every sub-frame so out of $n$ time slots first $w$ time slots in every sub-frame are reserved for type ' $T_{A}$ ' nodes (as represented in Figure 3, 'slot 1' to 'slot w' and Algorithm 1 [Lines 1-10]). Nodes in Category $T_{B}$, on the other hand, do not transmit in every subframe, rather, nodes in this category transmit once every few subframes and need to be arranged in order to maximize scheduling efficiency. As an example, see scheduling of nodes for 'Slot $\mathrm{w}+1$ ' in Figure 3. Two nodes are scheduled in this column, i.e. ' $\mathrm{Nw}+1$ ' and ' $\mathrm{Nw}+2$ '. Nodes $\mathrm{Nw}+1$ and $\mathrm{Nw}+2$ are scheduled alternatively as both of the nodes transmit in alternative sub-frames. To schedule these nodes, the scheduler first schedules both the nodes in two separate columns at first but since both the columns have vacant slots so one of the columns is circularly shifted and then merged together if they do not have overlapping schedules (which is true in this case). Note that the circular shift to the columns is limited by:

$$
L_{\text {shift }}=\left(T_{\text {deadline }} / \tau\right)-1
$$

In this case as time deadline of ' $\mathrm{Nw}+1$ ' and ' $\mathrm{Nw}+2$ ' is $2 \times \tau$, hence only one shift is performed. Similarly, in case of 'slot $\mathrm{w}+2$ ', three nodes are scheduled ' $\mathrm{Nw}+3$ ', ' $\mathrm{Nw}+4$ ' and ' $\mathrm{Nw}+5$ '. See that ' $\mathrm{Nw}+3$ ' has time deadline of $2 \tau$ whereas ' $\mathrm{Nw}+4$ ' and ' $\mathrm{Nw}+5$ ' have time deadline of $4 \tau$ respectively. First ' $\mathrm{Nw}+3$ ' is scheduled. Since ' $\mathrm{Nw}+4$ ' cannot be scheduled in same slot unless circularly shifted so the ' $\mathrm{Nw}+4$ ' was placed in the same column with one shift. The scheduler also schedules ' $\mathrm{Nw}+5$ ' in next columns and see by circularly shifting it up to ' $L_{\text {shift }}$ ' (see equation 4 ) and is merged with the same column with three shifts. Nodes belonging to category $T_{C}$ are scheduled in a similar way. After Nodes belonging to both $T_{B}$ and $T_{C}$ are separately scheduled, there could be some partially scheduled columns left. In Figure 3, columns referred as 'Slot p' and 'Slot s'still have unscheduled slots so scheduler merges the two by circularly shifting Slot $\mathrm{p}$ twice. Any remaining available free slots are filled with the schedule of nodes in category $T_{D}$. The final schedule is circulated to the nodes in the cluster before the data communication can start.

\section{Results and Discussion}

The performance of the scheduling algorithm is evaluated in terms of the schedule efficiency and energy efficiency. For the evaluation purposes the deadlines of the nodes are generated at random ranging from 10 milliseconds $(\mathrm{ms})$ to $400 \mathrm{~ms}$. As a reference, the desired update frequency/deadlines for selected control applications in industrial environments are presented in Table I. The time deadlines of the individual nodes in one of the evaluated scenarios is presented in Figure 4. Based on the defined range of deadlines, the values of $T_{s f}$ and $\tau$ are evaluated whereas 20 time slots are allocated in a sub-frame i.e. $k=$ 20. Furthermore, the number of generated information bits per sample, $f \_b i t s_{i}$, for node $i$ is assumed to take one time slot for communication.

Algorithm 1: Schedule formation for communication of heterogeneous sensor sampling in IWSNs.

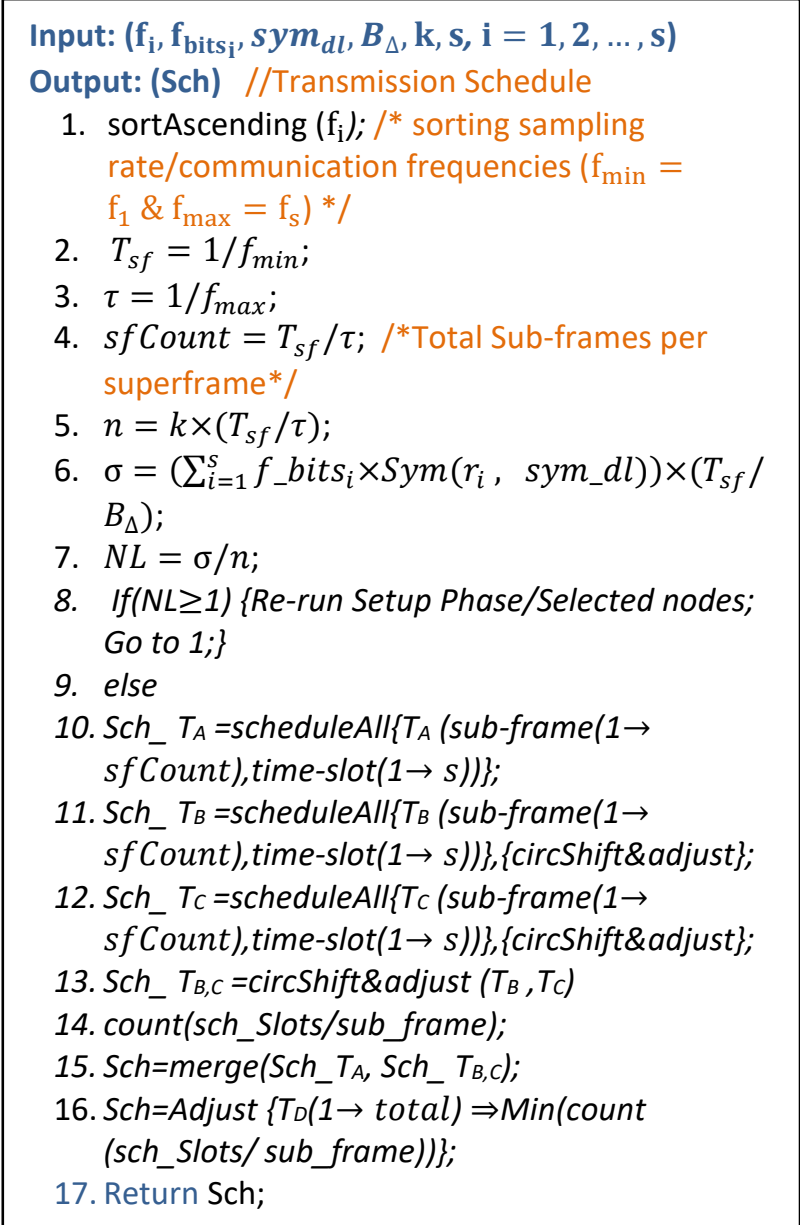

The performance of the scheduling algorithm is evaluated by increasing the load on the cluster-head by incrementing number of affiliated nodes. In Figure 5, the bar graphs represent the traffic load in percentage (right side y-axis) 
as the number of affiliated nodes are increased from 40 to 75 . In extreme cases (nodes $=75$ ), the traffic load reaches to 92.4\%. The error bars, represented in the second plot in Figure 5 (w.r.t left Y-axis) present the average number of scheduled slots per sub-frames, with error bar presenting deviation from the mean value for one complete superframe duration. It can be seen that for loads up to 92.4\% scheduler still manages to schedule nodes with diverse deadlines. Furthermore, the deviation in number of scheduled slots per sub-frame is well within the limits, ensuring deterministic performance due to minor variations in scheduled slots to shared slots ratio.

Since the proposed scheduling scheme offers a deterministic schedule, therefore, instead of listening to the beacon of each sub-frame (which would be considered as a superframe in traditional cases), the nodes with less frequent communications can choose to listen to the beacon of superframe. For instance, a node transmitting only once in the superframe does not need to listen to the beacon of each subframe. Rather, it will just listen to the beacon of superframe and then the beacon of subframe in which it is transmitting. Listening to the beacon of the sub-frame in which node is scheduled to transmit is used for fine tuning. The nodes can also rely on the superframe beacon only, but this may cause desynchronization and time jitter effects due to which sub-frame beacon of the relevant sub-frames is also considered.

Table 1. Delay and update requirements for industrial processes [16-18]

\begin{tabular}{lcc}
\hline \multicolumn{3}{l}{$\begin{array}{l}\text { Update frequency and deadlines for control applications in } \\
\text { IWSNs }\end{array}$} \\
\hline Application Type & $\begin{array}{l}\text { Update } \\
\text { Frequency }\end{array}$ & $\begin{array}{l}\text { Battery } \\
\text { lifetime }\end{array}$ \\
\hline \multicolumn{3}{c}{ Close Loop Control } \\
\hline Control valve [19, 20] & $10-500 \mathrm{~ms}$ & 5 years \\
Pressure sensor [19, 20] & $10-500 \mathrm{~ms}$ & 5 years \\
Temperature sensor [20] & $10-500 \mathrm{~ms}$ & 5 years \\
Variable speed drive [20] & $10-500 \mathrm{~ms}$ & 5 years \\
\hline \multicolumn{3}{c}{ Interlocking and Control } \\
\hline Proximity sensor [19, 20] & $10-250 \mathrm{~ms}$ & 5 years \\
Valve [19, 20] & $10-250 \mathrm{~ms}$ & 5 years \\
Machinery and tools & $10 \mathrm{~ms}$ & 3 years \\
Motion Control & $10 \mathrm{~ms}$ & 3 years \\
\hline
\end{tabular}

Since the active listening mode consumes a reasonable amount of energy, it is therefore, important to minimize the active listening period. In Figure 6 , the overall active listening period of the nodes in the proposed scheduling scheme as well as the traditional schemes is presented. It can be seen that with the deterministic schedule the overall active listening period during a superframe of duration $T_{s f}$ is notably reduced.

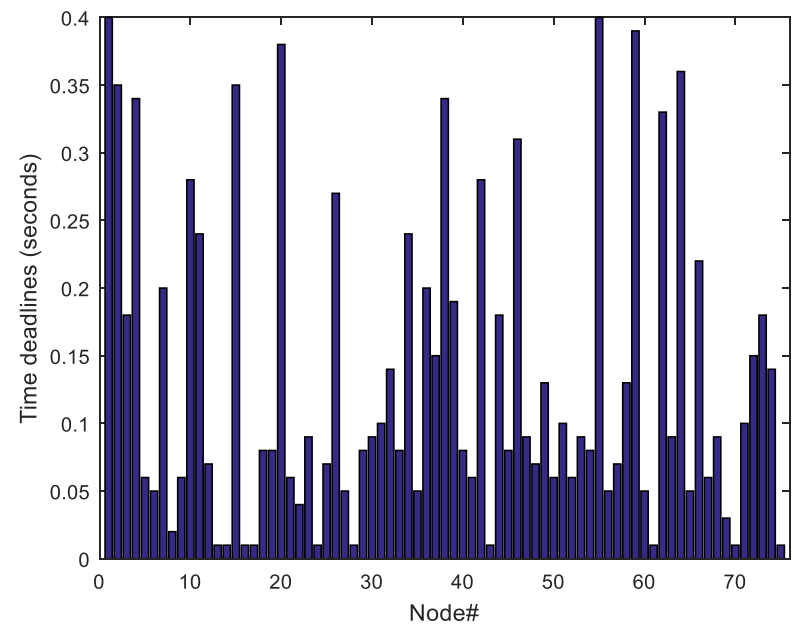

Figure 4. Time Deadlines of the Nodes affiliated with a cluster-head

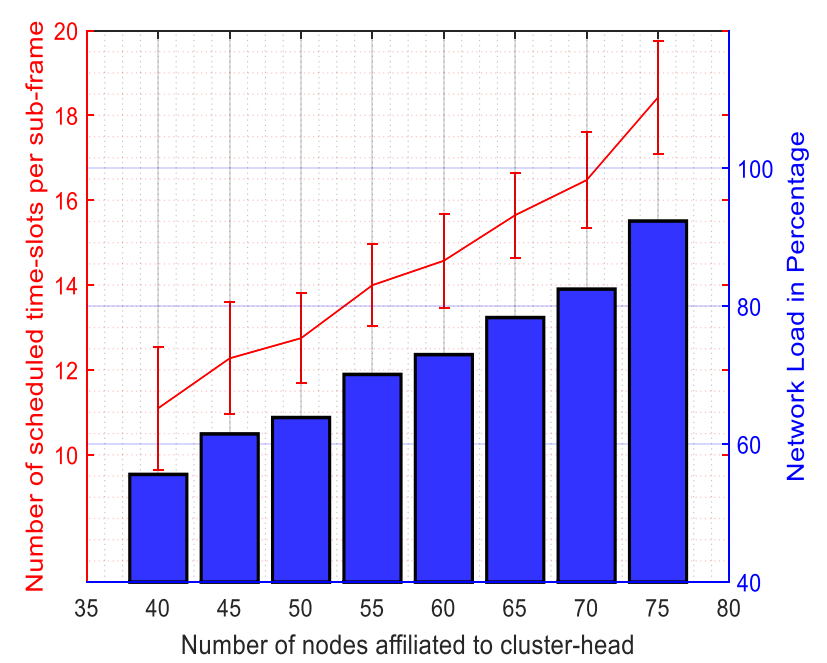

Figure 5. Scheduled slots per sub-frame as a function of Network load and number of affiliated nodes

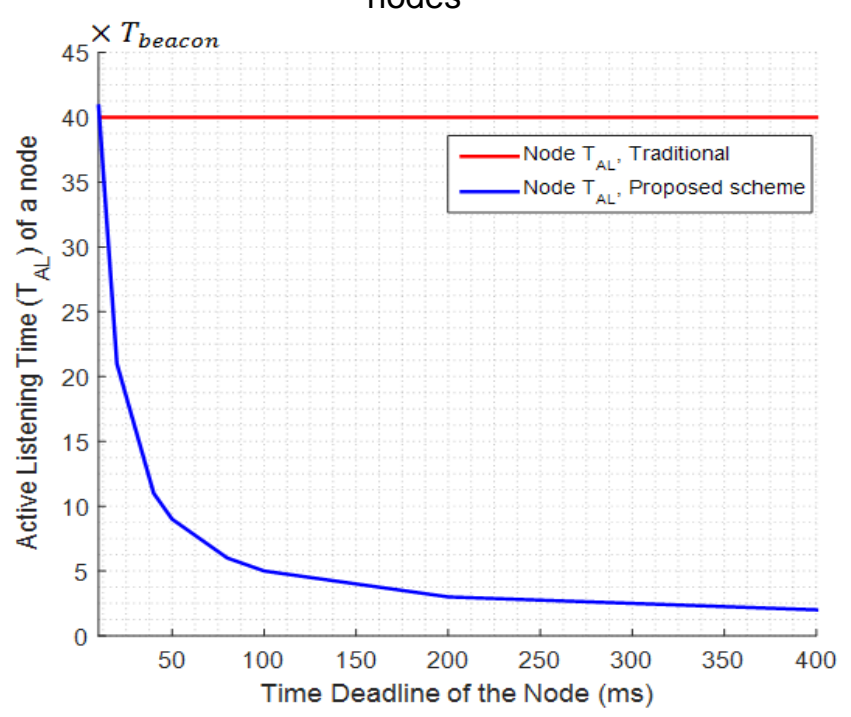

Figure 6. Active Listening time of a node in a superframe of duration $T_{s f}$ 


\section{Conclusion and Future Directives}

Industrial systems have diverse requirements and therefore nodes in IWSNs may have varying and desynchronized time deadlines. For such cases, a deterministic schedule formation for TDMA based communication in IWSNs, is a challenging task. In this paper, a scheduling algorithm is presented which offers a symmetric schedule for desynchronized node deadlines. The performance of the algorithm is evaluated based on the scheduled slots in each frame and scheduling ability of the presented scheme under high network loads. Furthermore, the generated schedule is used to improve the battery life of the wireless nodes by minimizing active listening periods in communications of these nodes.

The scheduling algorithm uses an approximation scheme to offer deterministic schedule. However, the work can further be extended by incorporating asynchronous communications in the schedule.

\section{References}

[1] P. Zand, S. Chatterjea, K. Das, and P. Havinga, "Wireless industrial monitoring and control networks: The journey so far and the road ahead," Journal of sensor and actuator networks, vol. 1, pp. 123-152, 2012.

[2] J. Akerberg, M. Gidlund, and M. Bjorkman, "Future research challenges in wireless sensor and actuator networks targeting industrial automation," in Industrial Informatics (INDIN), 2011 9th IEEE International Conference on, 2011, pp. 410-415.

[3] Z. Liu, W. Zhang, S. Lin and T. Q. S. Quek, "Heterogeneous Sensor Data Fusion By Deep Multimodal Encoding," in IEEE Journal of Selected Topics in Signal Processing, vol. 11, no. 3, pp. 479-491, April 2017.

[4] Z. Meng, L. Junru, L. Wei, and Y. Haibin, "A priority-aware frequency domain polling MAC protocol for OFDMA-based networks in cyber-physical systems," Automatica Sinica, IEEE/CAA Journal of, vol. 2, pp. 412-421, 2015.

[5] D. De Guglielmo, F. Restuccia, G. Anastasi, M. Conti and S. K. Das, "Accurate and Efficient Modeling of 802.15.4 Unslotted CSMA/CA through Event Chains Computation," in IEEE Transactions on Mobile Computing, vol. 15, no. 12, pp. 2954-2968, Dec. 12016.

[6] "IEEE Standard for Local and metropolitan area networks--Part 15.4: Low-Rate Wireless Personal Area Networks (LR-WPANs)," IEEE Std 802.15.4-2011 (Revision of IEEE Std 802.15.4-2006), pp. 1-314, 2011.

[7] K. Ashrafuzzaman, "Energy and Throughput Optimal Operating Region in Slotted CSMA/CA Based WSN," in IEEE Communications Letters, vol. 16, no. 9, pp. 1524-1527, September 2012.

[8] S. Wei, Z. Tingting, F. Barac, and M. Gidlund, "PriorityMAC: A Priority-Enhanced MAC Protocol for Critical Traffic in Industrial Wireless Sensor and Actuator Networks," Industrial Informatics, IEEE Transactions on, vol. 10, pp. 824-835, 2014.

[9] Y. Dong, X. Youzhi, W. Hongchao, Z. Tao, Z. Hao, Z. Hongke, et al., "Assignment of Segmented Slots Enabling Reliable Real-Time Transmission in Industrial Wireless Sensor Networks," Industrial Electronics, IEEE Transactions on, vol. 62, pp. 3966-3977, 2015.

[10] D. S. Waghole and V. S. Deshpande, "Analyzing the QoS using CSMA and TDMA protocols for wireless sensor networks," International Conference for Convergence for Technology-2014, Pune, 2014, pp. 1-5.

[11] "IEEE Standard for Local and metropolitan area networks--Part 15.4: Low-Rate Wireless Personal Area Networks (LR-WPANs) Amendment 1: MAC sublayer," IEEE Std 802.15.4e-2012 (Amendment to IEEE Std 802.15.4-2011), pp. 1-225, 2012.
[12] S. C. Ergen and P. Varaiya, "TDMA scheduling algorithms for wireless sensor networks," Wireless Networks, vol. 16, pp. 985-997, 2010.

[13] W. Shen, T. Zhang, M. Gidlund, and F. Dobslaw, "SAS-TDMA: A source aware scheduling algorithm for real-time communication in industrial wireless sensor networks," Wireless networks, vol. 19, pp. 1155-1170, 2013

[14] M. Yan, K.-Y. Lam, S. Han, E. Chan, Q. Chen, P. Fan, et al., "Hypergraph-based data link layer scheduling for reliable packet delivery in wireless sensing and control networks with end-to-end delay constraints," Information Sciences, vol. 278, pp. 34-55, 2014.

[15] F. Dobslaw, T. Zhang, and M. Gidlund, "End-to-End Reliabilityaware Scheduling for Wireless Sensor Networks," Industrial Informatics, IEEE Transactions on, vol. PP, pp. 1-1, 2014.

[16] T. Zheng, M. Gidlund, and J. Akerberg, "WirArb: A New MAC Protocol for Time Critical Industrial Wireless Sensor Network Applications," Sensors Journal, IEEE, vol. PP, pp. 1-1, 2015.

[17] J. Akerberg, M. Gidlund, and M. Bjorkman, "Future research challenges in wireless sensor and actuator networks targeting industrial automation," in Industrial Informatics (INDIN), 2011 9th IEEE International Conference on, 2011, pp. 410-415.

[18] P. Neumann, "Communication in industrial automation-What is going on?," Control Engineering Practice, vol. 15, pp. 1332-1347, 2007.

[19] J. Xia, C. Zhang, R. Bai, and L. Xue, "Real-time and reliability analysis of time-triggered CAN-bus," Chinese Journal of Aeronautics, vol. 26, pp. 171-178, 2013.

[20] D. Christin, P. S. Mogre, and M. Hollick, "Survey on wireless sensor network technologies for industrial automation: The security and quality of service perspectives," Future Internet, vol. 2, pp. 96-125, 2010. 